\title{
Legitimizing Bitcoin as a Currency and Store of Value: Using Discrete Monetary Units to Consolidate Value and Drive Market Growth
}

\author{
Chad Albrecht, ${ }^{\dagger}$ Steven Hawkins, ${ }^{\dagger}$ Kristopher McKay Duffin ${ }^{\S}$
}

\begin{abstract}
Cryptocurrency, and especially Bitcoin, has struggled to gain recognition as a legitimate currency from governments, financial institutions, and consumers. ${ }^{1}$ This has occurred because many analysts and consumers believe that Bitcoin is not a stable and consistent store of value, a unit of measurement, or a medium of exchange. ${ }^{2}$ One way to overcome this challenge is for Bitcoin to be used as both a currency and store of value by a greater percentage of the world's population. ${ }^{3}$ This paper seeks to identify how a change in Bitcoin's monetary measurement (or denomination) can more easily facilitate Bitcoin transactions to increase its use. Specifically, we posit that applying whole number bias theory, from the cognitive psychology and mathematics fields, to Bitcoin's unit of measurement will allow the value of Bitcoin to be referenced in smaller and easier to understand units with fewer numbers after the decimal point—such as the "Bit" or the "Satoshi." In the process, the use of Bitcoin will include more whole numbers and allow the general public to more easily assign value to Bitcoin in day-to-day transactions.
\end{abstract}

\section{Introduction}

According to Satoshi Nakamoto's original white paper on Bitcoin, cryptocurrency is a nascent form of digital currency, which facilitates direct peer-to-peer transactions without the use of a third party or financial intermediary. Bitcoin transactions are validated using a blockchain, Bitcoin's underlying technology. A blockchain is a public ledger that details and verifies all previous transactions. The Bitcoin blockchain is compiled by "mining" for bitcoins in a combinatorial space. ${ }^{4}$ When individuals mine for Bitcoin, they are compiling transactions within its blockchain. This process prevents the community of users from double spending and approves or verifies all transactions. These transactions are facilitated over the internet and allow the transfer of value throughout the world.

Bitcoin has often been compared to gold as the function and scarcity between the assets are similar. As more bitcoins are mined, for example, the harder it becomes to mine additional bitcoins. The pool of available bitcoins halves every 210 blocks (or roughly every four years) until all bitcoins have essentially been mined. Bitcoin, as a monetary unit, functions as the basic measurement of the currency. Over the last few years, the price of Bitcoin has fluctuated and, in the process, experienced significant volatility which limits consumers from using the

† C. Albrecht (chad.albrecht@usu.edu) is a Full Professor of Strategy \& Director of MBA Programs at the Jon. M. Huntsman School of Business at Utah State University.

* S. Hawkins is an Assistant Professor of Accounting at the Dixie L. Leavitt School of Business at Southern Utah University,

$\S \mathrm{K}$. McKay Duffin is a Research Associate in the Jon M. Huntsman School of Business at Utah State University. 
currency in day-to-day financial transactions. This friction, along with the lack of user adoption has, at times, led to Bitcoin being used more for speculative investment than as a currency. ${ }^{5}$

In order for Bitcoin to function as a currency in day-to-day transactions, we posit that Bitcoin's monetary unit of analysis (or denomination) must become more simple to understand and include fewer numbers after the decimal place during everyday transactions. In other words, we suggest that Bitcoin, in its current form, is too difficult for the average consumer to assign value to, as using Bitcoin in day-to-day transactions results in too many decimal or fractional parts of the Bitcoin. The fractional part, or numerical part, of a number that occurs after the decimal is often referred to in mathematics as the mantissa. In this paper, we suggest that when applied to Bitcoin, the mantissa, or the part of the logarithm that follows the decimal point is, in its current form, too large for day-to-day and micro transactions. In the cognitive psychology field, individual preference towards whole numbers over decimals is often referred to as the whole number bias theory (or natural number bias theory). This theory has been studied and exhibited in both adults and children.

In sum, when applied to Bitcoin, whole number bias theory suggests that very few individuals would want to engage in commerce with a numerical value system that engages multiple digits after the decimal place. In other words, under whole number bias theory, most consumers would not want to spend .00091232 Bitcoins for a cup of coffee. Rather, because consumers prefer whole numbers with limited decimal places, most consumers would prefer engaging in an easier-to-understand monetary system such as the U.S. Dollar, where a cup of coffee would cost just $\$ 5.50$.

In this scenario, for most consumers, the unitary measurement of Bitcoin becomes too complicated with 8 decimal places, creating confusion in assigning value to the transaction. Similarly, most individuals would prefer to have $\$ 2,500$ in their checking account compared to .33219732 Bitcoins. In each of these scenarios, the lack of whole numbers is perceived by consumers to be too small and complicated and the value too nominal. When compared to most major currencies around the world, including the US Dollar, the British Pound, the Euro, the Japanese Yen, the Mexican Peso, or the Chinese Renminbi we see that Bitcoin is the only currency where one monetary base unit is so valuable (from $\$ 3,000$ to $\$ 20,000$ in 2018 alone) and where the mantissa will commonly have six, seven, or even eight digits after the decimal. In sum, the pure length of Bitcoin's mantissa often creates cognitive dissonance in the individual, creating a natural tendency for consumers to conduct day-to-day transactions in currencies that employ whole numbers.

Fortunately, as discussed in various papers, there are a number of measurement units that make up a single Bitcoin. ${ }^{6}$ One such monetary unit, for example, is the "Bit," which equates to roughly $1 / 1,000,000$ of a single Bitcoin. Similarly, a secondary monetary unit is the "Satoshi" which equates to roughly $1 / 100,000,000$ of a Bitcoin.

The idea to refer to Bitcoin in smaller monetary units has existed for some time and was first proposed on various online forums going as far back as 2012. While some exchanges and wallets currently reference, or co-list, smaller monetary units of Bitcoin, it is not the norm within the industry. One such wallet, The Wallet of Satoshi, was developed in Australia and uses the lightning network. Interestingly, the Wallet of Satoshi co-lists transactions in both satoshis and dollars, providing consumers with valuable information and allowing them to easily assign value to a transaction. In the process, the wallet limits the use of any decimal values in their 
reporting procedures. Similarly, Bitpay, a large Bitcoin payment processing company, has also recently changed their wallet to include both 'Bits' and 'U.S. Dollars' further simplifying the payment and value system for those unfamiliar with cryptocurrency. Because of the importance of smaller Bitcoin denominations in daily transactions, a number of online forums have begun proposing and discussing potential symbols for the Satoshi in hopes that the Satoshi will become more well-known and used within the Bitcoin community.

While some consumers who enact in Bitcoin transactions use smaller divisible units of Bitcoin (such as bits or satoshis), most of the general public is unaware of the fact that they can buy and spend less than one Bitcoin, limiting the adoption and use of Bitcoin by the general public. ${ }^{7}$

The following sections will investigate and further explain whole number bias theory, Bitcoin's challenges as a currency, consumer perceptions of Bitcoin, the history of proposing the use of discrete units, and how Bitcoin's standard monetary unit can be changed.

\section{What Is Whole Number Bias Theory?}

As was said earlier, whole number bias theory suggests that individuals have a natural tendency or preference for using whole numbers over decimals when working with numbers. ${ }^{8}$ For example, a comprehensive study by DeWolf suggests that in relational modeling, consumers display a clear preference for fractions over decimals. ${ }^{9}$ Their reasoning for this phenomenon is that fractions represent a two-dimensional relationship between discrete quantities, while decimals offer a one-dimensional relationship involving proportions. Relational modeling in this context refers to the act of comparing the relationships between two sets of objects or values. In the study, participants ordered fractional and rational numbers from the lowest magnitude to the greatest magnitude on a number line, or were asked to assess the magnitude of numbers already on a number line. DeWolf found that when the fractions being compared were placed on the number line, that as the fractions became closer together, their magnitudes became more difficult to assess.

Whole number bias is also present in children. In a study by Roell and Virarogue, children were asked to compare the magnitudes of a group of rational numbers. ${ }^{10}$ In the study, children mistakenly attributed numbers with more digits when compared to those numbers with fewer digits. The researchers concluded that the children demonstrated whole number bias as a result of their own conceptual frameworks that had already been established with regards to the value of whole numbers.

Under whole number bias theory, a whole number with a greater number of digits will always have a greater magnitude than a whole number with fewer digits. However, with rational numbers, the opposite is true. An increased number of digits after the decimal point does not necessarily result in a proportional increase in magnitude. ${ }^{11}$

Research has also suggested that whole number bias occurs in various cultures. For example, Lai and Wong suggest the presence of whole number bias in Chinese children, demonstrating that whole number bias emerges independent of different mathematical conceptualizations. ${ }^{12}$ For example, the Chinese language uses explicit and direct language when describing both large 
and rational numbers. ${ }^{13}$ And, although research suggests that Chinese children have a better understanding of rational numbers when compared to children from other countries, every child studied in the research, including Chinese children, still presented whole number bias in their behavior to varying degrees.

It has been suggested that whole number bias is generated through conceptual restructuring. ${ }^{14}$ When children transition from learning about whole numbers to rational numbers, they must form a new conceptual framework. The difficulty to switch from wellestablished conceptual frameworks to new frameworks persists into adulthood. While adults can shift from one conceptual framework to another framework more efficiently than children, they are often unwilling to do so. ${ }^{15}$ Because adults prefer to use conceptual frameworks they are familiar with, it becomes increasingly important that Bitcoin and other cryptocurrencies keep within commonly established monetary frameworks that include whole numbers with limited decimal places.

\section{Bitcoin: Challenges as a Currency}

Bitcoin itself is defined as a decentralized unit of exchange that facilitates digital peer-to-peer transactions. However, most individuals agree that Bitcoin is not recognized as having intrinsic value other than the trust that individuals place in it. As such, in its short history, Bitcoin has had little influence on the policies of central banks and existing "fiat" currencies. ${ }^{16}$

It should be noted that even with the various challenges that Bitcoin is facing, the use of Bitcoin by consumers is increasing, and a growing number of businesses accept cryptocurrencies for various transactions. However, at the same time, most governmental agencies, including numerous official regulatory government bodies such as the SEC, do not recognize cryptocurrencies, including Bitcoin, as legitimate currencies. ${ }^{17}$

One of Bitcoin's most frequent criticisms is that Bitcoin's price is manipulated by various stakeholders. This susceptibility further prevents Bitcoin from gaining legitimacy from regulators, consumers, and others. To increase credibility, a number of financial and Bitcoinfocused institutions have attempted to gain recognition from the SEC, as well as obtain SEC approval for numerous Bitcoin derivative instruments, including a Bitcoin ETF (electronic traded fund). However, up until this point, the SEC has denied all ETF applications and requests. ${ }^{17}$ This lack of clarity and rules by regulators has further limited Bitcoin's growth and led some to refer to Bitcoin and other cryptocurrencies as 'The Wild West'.

The risks involving Bitcoin extend beyond the scope of price manipulation. Centralized exchanges that exchange traditional currency for Bitcoin have the tendency to be targeted with hacks and other crimes. ${ }^{18}$ This results in vast amounts of Bitcoin being stolen. These types of incidents have occurred numerous times in the past and will, unfortunately, likely continue into the future. It should be noted, however, that some exchanges such as Gemini and Coinbase have recently begun to insure Bitcoin deposits on their exchanges. This insurance significantly decreases the perceived risk of working with, and using, such exchanges.

According to Gandal and Hamrick, the ecosystem surrounding Bitcoin itself is often manipulated to artificially increase the price of Bitcoin. ${ }^{19}$ In their study, they found that Bitcoin 
prices rise significantly on days where suspicious transactions take place. While Bitcoin and its blockchain are theoretically designed to facilitate anonymous transactions, Gandal and Hamrick also found that cryptocurrency exchanges such as Mt. Gox recorded all transactions and linked them to specific user profiles. In 2014, this list of transactions by Mt. Gox was leaked to the public. A final factor that has discouraged large scale investment in Bitcoin and other cryptocurrencies is the fact that traditional valuation methods often cannot be applied to evaluate the value of cryptocurrency.

\section{History of Proposing the Use of the Bit or Satoshi}

As was stated earlier, the idea of using bits, or satoshis to replace bitcoins as a reference to the Bitcoin monetary value system has existed within the cryptocurrency community for some time. Such opinions are often circulated in online forums such as Reddit (a popular website that hosts threads on various topics and includes discussions, posts, forums, and feeds). Reddit allows users to organically circulate content and writing with others with similar interests. Within the Reddit community, the use of the Satoshi continues to be advocated-especially as the value of Bitcoin itself has risen.

Other users, however, have proposed the use of the MicroBitcoin. One Reddit user, $\mathrm{u} /$ brcreeker, for example, posted a thread titled, "A case for the MicroBitcoin (uBTC) or Sato Bitcoin (sBTC)," where the benefits of using Bits for everyday transactions is discussed. ${ }^{20}$ The main argument presented in the thread is that, as the value of the Bitcoin reaches thousands of U.S. dollars, the MicroBit has the potential to reach parity with the United States cent. Currently, depending on Bitcoin's value at the time, the MicroBit is approaching parity with currencies from a number of countries including Mexico, Kenya, and Israel. If the Bit reaches parity with the smallest units of most world currencies over time, then using the Bit will increase user adoption and incentivize the use of Bitcoin as a currency.

In a thread titled, "Isn't it about time we standardized mBTC as the official unit of Bitcoin?" by Reddit user $\mathrm{u} /$ armysatoru, the author explores the idea of using smaller divisible units for everyday transactions using Bitcoin. ${ }^{21}$ The argument presented in the thread encompasses the idea of using the unit mBTC as the standard unit of exchange to encourage everyday users to become involved in the cryptocurrency market. The author argues that user participation is limited due to the misconception that one would have to buy one whole Bitcoin in order to use the cryptocurrency, when smaller fractions of a Bitcoin can be purchased. Using smaller divisible units such as the $\mathrm{mBTC}$ is important because it would increase user adoption by allowing people to utilize Bitcoin for everyday financial transactions. Since there is no centralized body that controls Bitcoin or other cryptocurrencies, the standard unit of the Bitcoin, while influenced by many factors, must be changed by the community of users itself. 


\section{How to Change Bitcoin's Monetary Unit}

Since Bitcoin is a decentralized framework, there are no official governing bodies or authoritative decision makers within its blockchain. As such, integrating a change to the divisible unit of Bitcoin may prove difficult to implement. However, because a number of institutions in the space have immense influence, a coordinated effort to change Bitcoin's monetary unit is still possible. Exchanges, developers, and wallets are probably in the best position to change Bitcoin's monetary unit of measurement.

Because Bitcoin exchanges broker and report the exchange rate and values for Bitcoin, the exchanges have a large influence on how bitcoin is reported and measured-including its monetary unit of analysis. While hundreds of exchanges exist, many of them are unregulated. Because the value and exchange rate of Bitcoin is volatile, many exchanges list different values for Bitcoin at any given time. There are also several websites that compile and report Bitcoin's price. Probably the most popular website reporting exchange data and prices is CoinMarketCap. ${ }^{22}$ While there are hundreds of exchanges throughout the world, we posit that if several of the most influential exchanges were to change their reporting and trading process to reflect smaller units, then most other exchanges and wallets would follow suit. For example, some of the most influential exchanges that could potentially lead this effort include Binance, Square, Coinbase, BitMex, Gemini, Kraken, Bittrex, Huobi, Poloniex, GDAX, Liquid, Coinfield, OKCoin, Cexio, Ind.Reserve, BitFlyer, Bitstamp, ItBit, CEX.IO, Robinhood, Circle, OKEx, Luno, Deribit, FTX, Ethfinex, Gate, Bakkt, Bitso, Belfrics, LGO, Delta, IDEX, Xfutures, Blocktrade, Vindax, Jex, Yobit, Livecoin, and Bitfinex.

While the current price of Bitcoin is extremely volatile (especially when compared to traditional assets such as stocks, bonds and gold), Li and Wang suggest that Bitcoin exchange rates will stabilize over time and become influenced by traditional economic variables rather than speculative trading. ${ }^{23}$ In their research, they suggest that as the mining process becomes easier with time and technology, Bitcoin mining will become more decentralized and limit the influence of centralized mining pools and companies.

In addition to exchanges, online wallets have a large influence within the Bitcoin community. Gutoski and Stebila state that Bitcoin wallets are online connections to the private keys of Bitcoin. ${ }^{24} \mathrm{~A}$ master key allows one to unlock the wallet. Every Bitcoin has a public and a private key. When a user has both, they are able to spend the Bitcoin. Online wallet services have a large impact on Bitcoin use because they are the main storage method for the vast majority of Bitcoin users. Simply put, if wallets change the way they report and store Bitcoin, it will have a large influence on the overall community.

Since Bitcoin is decentralized, any change to the main divisible unit must come from the entire community of users. One suggestion to implement the use of the mBTC was proposed in the opinion article, "It's Time to Change the Way We Measure Bitcoin" on the website bitcoin.com. ${ }^{25}$ In the article, the author proposes hosting a "B-day," where all users and exchanges switch to a smaller unit of measurement instead of using whole Bitcoins. While there is still debate as to whether the unit of analysis should change to Bits, MiliBits, or Satoshis, if this movement were to gain enough social support, the movement would likely change how Bitcoin is perceived by the public. In the process, Bitcoin would likely transition from a speculative asset and store of value to also becoming a widely-used currency, further facilitating the use of Bitcoin in day-to-day transactions. 


\section{Conclusion}

This paper has explored how to legitimize Bitcoin as a currency by using smaller discrete units to allow the use of whole numbers, consolidate value and drive market growth. Bitcoin was first introduced as a peer-to-peer decentralized currency. While Bitcoin has the potential to be treated like, and even replace certain fiat currencies, Bitcoin has not realized its full potential nor experienced widespread adoption by consumers and others.

To further highlight the importance of using whole numbers, in the paper, we introduced the whole number bias theory from the cognitive psychology and mathematics fields and then applied that theory to the Bitcoin framework. Whole number bias, or natural number bias, suggests that individuals prefer whole numbers over decimals or fractions. Whole number bias theory provides an important perspective on Bitcoin. With most small transactions nearing the sixth, seventh or even eighth decimal point, Bitcoin's use in day-to-day and micro transactions is limited in its current form.

In order to promote Bitcoin and facilitate Bitcoin adoption and value, we posit that Bitcoin's monetary unit of analysis, or denomination, should be switched to smaller increments such as the Bit, MiliBit, or Satoshi. By so doing, the Bitcoin framework would encourage consumers to make everyday purchases with whole numbers instead of fractions of a bitcoin or an extended number of decimals. In the process, we believe that Bitcoin's ease of use and adoption would increase significantly, further increasing Bitcoin's use as a stable and consistent store of value, unit of measurement, and medium of exchange.

\section{Author Contributions}

C.A. contributed towards idea generation, the outline of the paper, theory development, topic refinement, review and analysis, argument formulation, revisions, and the abstract. S.H. contributed towards idea generation, theory development, paper organization and layout, introduction and drafting of the initial body, conclusion, and literature review. M.D. contributed towards writing, grammar, references, theory development, paper organization and layout, introduction and drafting of the initial body, conclusion, and literature review.

\section{Notes and References}

${ }^{1}$ Moore, E., Christin, N. "Beware the Middleman: Empirical Analysis of Bitcoin-Exchange Risk.” In Sadeghi, A. R. (Ed.) Financial Cryptography and Data Security FD 2013. Berlin, Heidelberg: Springer 25-33 (2013) https://doi .org/10 .1007/978-3-642-398841_3. 
${ }^{2}$ Kubat, M. "Virtual Currency Bitcoin in the Scope of Money Definition and Store of Value." Procedia Economics and Finance 30 409-416 (2015) https : / doi . org/10.1016/ S2212-5671 (15) 01308-8.

${ }^{3}$ Glaser, F., Zimmermann, K., Haferkorn, M., Weber, M. C., Siering, M. "Bitcoin - Asset or Currency? Revealing Users' Hidden Intentions." Twenty Second European Conference on Information Systems (2014). Available at SSRN: https: / / papers.ssrn.com/sol3/ papers.cfm?abstract_id=2425247.

${ }^{4}$ Nakamoto, S. "Bitcoin: A Peer-to-Peer Electronic Cash System.” (2008) (accessed 30 January 2020) https://bitcoin.org/bitcoin.pdf.

${ }^{5}$ Cheah, E., Fry, J. "Speculative bubbles in Bitcoin markets? An empirical investigation into the fundamental value of Bitcoin." Economics Letters 130 32-36 (2015)

https://doi.org/10.1016/j.econlet.2015.02.029.

${ }^{6}$ Madise, S. "Back to the Future: Evolving Forms of Money." SSRN (2015)

http://dx.doi.org/10.2139/ssrn.2622080.

${ }^{7}$ Luther, J. "Bitcoin and the Future of Digital Payments." The Independent Review 20 397-404 (2016) http://www.independent.org/pdf/tir/tir_20_03_12_luther.pdf.

${ }^{8}$ Vamvakoussi, X. "Naturally Biased? In Search for Reaction Time Evidence for a Natural Number Bias in Adults." The Journal of Mathematical Behavior 31.3 344-355 (2012) https://doi.org/10.1016/j.jmathb.2012.02.001.

${ }^{9}$ DeWolf, M., Vosniadou, S. "The Representation of Fraction Magnitudes and the Whole Number Bias Reconsidered." Learning and Instruction 37 39-49 (2015) https : / do i . org/ $10.1016 / j$. learninstruc.2014.07.002.

${ }^{10}$ Roell, M., Viarouge, A., Houdé, O., Borst, H. "Inhibition of the Whole Number Bias in Decimal Number Comparison: A Developmental Negative Priming Study." Journal of Experimental Child Psychology 77 240-24 (2019) https: / / doi .org/10.1016/j .jecp .2018 .08 .010 .

${ }^{11}$ DeWolf, M., Bassok, M. "From Rational Numbers to Algebra: Separable Contributions of Decimal Magnitude and Relational Understanding of Fractions." Journal of Experimental Child Psychology 133 72-84 (2015) https: / / doi.org/10.1016/j .jecp.2015.01.013.

${ }^{12}$ Lai, M., Wong, J. "Revisiting Decimal Misconceptions from a New Perspective: The Significance of Whole Number Bias in the Chinese Culture." The Journal of Mathematical Behavior 47 96-108 (2017) https: / doi.org/10.1016/j.jmathb.2017.07.00. 
${ }^{13}$ Miller, K., Major, S. M., Shu, H., Zhang, H. “Original Knowledge: Number Names and Number Concepts in Chinese and English." Canadian Journal of Experimental Psychology. 54.2 129-139 (2000) https://doi.org/10.1037/h0087335.

${ }^{14} \mathrm{Ni}$, Y., Zhou, Y. "Teaching and Learning Fraction and Rational Numbers: The Origins and Implications of Whole Number Bias." Educational Psychologist 40 (2005)

https://doi.org/10.1207/s15326985ep4001_3.

${ }^{15}$ Obersteiner, A., Van Dooren, W., Van Hoof, J., Verschaffel, L. "The Natural Number Bias and Magnitude Representation in Fraction Comparison by Expert Mathematicians." Learning and Instruction 28 64-72 (2013) https: / / doi.org/10.1016/j.learninstruc.2013 .05 .003 .

${ }^{16}$ Baur, D., Hong, K. "Bitcoin: Medium of Exchange or Speculative Assets?" Journal of International Financial Markets, Institutions, and Money 54 177-189 (2018)

https://doi.org/10.1016/j.intfin.2017.12.004.

${ }^{17}$ Fields, B. "Securities and Exchange Commission (Release No. 34-83723; File No. SRBatsBZX-2016-30)." Securities and Exchange Commission (2018) https: / /www . sec.gov /rules/other/2018/34-83723.pdf.

${ }^{18}$ Vasek, M., Thornton, M., Moore, T. "Empirical Analysis of Denial-of-Service Attacks in the Bitcoin Ecosystem.” In Böhme, R., Brenner, M., Moore, T., Smith, M. (Eds.) Financial Cryptography and Data Security. FC 2014. 57-71 (2014) https://doi .org/10.1007/9783-662-44774-1_5.

${ }^{19}$ Gandal, N., Hamrick, J. T., Moore, T., Oberman, T. "Price Manipulation in the Bitcoin Ecosystem." Journal of Monetary Economics 95 86-96 (2018) https: //doi .org/10.1016 /j.jmoneco.2017.12.004.

${ }^{20} \mathrm{u}$ /brcreeker "r/Bitcoin: A Case for the Microbitcoin (uBTC) Satobitcoin (sBTC)" Reddit. (accessed 12 February 2019) https: / / www. reddit.com/r/Bitcoin/comments/1rg2vw /a_case_for_the_microbitcoin_ubtc_satobitcoin_sbtc/.

${ }^{21} \mathrm{u}$ /armysatoru "r/Bitcoin: Isn't It About Time We Standardized mBTC as the Official Unit of Bitcoin?" Reddit. (accessed 12 February 2019) https://www. reddit.com/r/Bitcoin /comments/7alpsj/isnt_it_about_time_we_standardized_mbtc_as_the/.

${ }^{22}$ No Author. "Top 100 Cryptocurrencies by Market Capitalization." CoinMarketCap (accessed 10 February 2019) https: / / coinmarketcap.com/.

${ }^{23} \mathrm{Li}, \mathrm{X}$., Wang, C. "The Technology and Economic Determinants of Cryptocurrency Exchange Rates: The Case of Bitcoin.” Decision Support Systems 95 49-60 (2017) https://doi.org/10.1016/j.dss.2016.12.001. 
${ }^{24}$ Gutoski, G., Stebila, D. "Hierarchical Deterministic Bitcoin Wallets that Tolerate Key Leakage." In Böhme, R., Okamoto, T. (Eds.) Financial Cryptography and Data Security. FC 2015. 497-504 (2015) https://doi.org/10.1007/978-3-662-47854-7_31.

${ }^{25}$ Sedgwick, K. "It's Time to Change the Way We Measure Bitcoin.” Bitcoin.com (accessed 16 December 2018) https: //news.bitcoin.com/its-time-to-change-the-way-wemeasure-bitcoin/.

(cc)BY ULLS
Articles in this journal are licensed under a Creative Commons Attribution 4.0 License.

Ledger is published by the University Library System of the University of Pittsburgh as part of its D-Scribe Digital Publishing Program and is cosponsored by the University of Pittsburgh Press.

10

ledgerjournal.org 